\title{
A Game Theory Approach for DVB-RCS Resource Allocation
}

\author{
Enrico Del Re*, Gherardo Gorni ${ }^{\dagger}$, Luca Simone Ronga ${ }^{\dagger}$, María Ángeles Vázquez Castro ${ }^{\ddagger}$ \\ *Department of Electronics and Telecommunications (DET), University of Florence, Italy \\ Email: enrico.delre@unifi.it
}

${ }^{\dagger}$ National Inter-University Consortium for Telecommunications (CNIT), University of Florence, Italy

Email: \{gherardo.gorni, luca.ronga\}@ cnit.it

\begin{abstract}
†Telecommunications and System Engineering Department (TES), Universitat Autònoma de Barcelona, Spain
Email: angeles.vazquez@uab.es
\end{abstract}

\begin{abstract}
A DVB-RCS based, hierarchical satellite network model is presented. The network is constituted by two levels for the allocation of the resources, i.e. beam level and terminal level. For each level will be studied two differents approaches, in particular the second level resource allocation has been developed according two different game theoretical approaches, based on both non-cooperative and cooperative game theory.

Index Terms-satellite communications, DVB-RCS, resource allocation, Game Theory.
\end{abstract}

\section{INTRODUCTION}

The new generation satellite standards, DVB-S2, [1], and DVB-RCS, [2], are meant to deliver multimedia contents over time-varying channels in the Ka frequency band or above. In this portion of the frequency spectrum, the communication are particularly sensitive to atmosperical events such as rain and clouds. It has been verified for these standards, [3], that in case of hostile atmospherical events, interactive TCP based elastic connections show a fast decrease of data throughput leading to an out-of-service period for the whole duration of the event.

Our idea to deal with this issue, it is to perform a distributed resource allocation between satellite terminals using a game theoretical approach. Game theory is a mathematical tool that allows to model interactions between subjects with conflicting objectives. Game theory has already been adopted for the rate control of information sources in high-speed networks, [4]. It has been applied also in wireless networks to perform a distributed medium access control, [5]. The reasons beacuse game theory is appropriate for communications contests is due both to its distributed nature and to its high scalability, as it has been argumented in [6].

In the next section the system architecture will be introduced. In section III the first level resource allocation methods will be described. The game theory approaches for the second level of the hierarchical system are presented in detail in section IV. In section $\mathrm{V}$ we examine the results of the simulations and we compare, for each level, the methods considered. Finally our conclusions will be discussed.

\section{The SAtellite Resource Hierarchy}

We consider as reference architecture a DVB-RCS hierarchical satellite network, constituted by two levels as shown in figure 1. In the first level the global resource to access the uplink channel is assigned to beams constituting the footprint of the satellite on the ground. A beam achieves a part of the resource accordingly an algorithm will be specified in section III. In the second level of the hierarchy, the resource formerly assigned to a beam is shared between the satellite stations located in that beam. These satellite stations can be thought both as independent terminals or as gateway providing Internet access to terminals in a subnet. These stations compete for the shared resource using a game theoretical approach that will be presented in section IV. The resource, that is shared in both levels of the system, is the bandwidth.

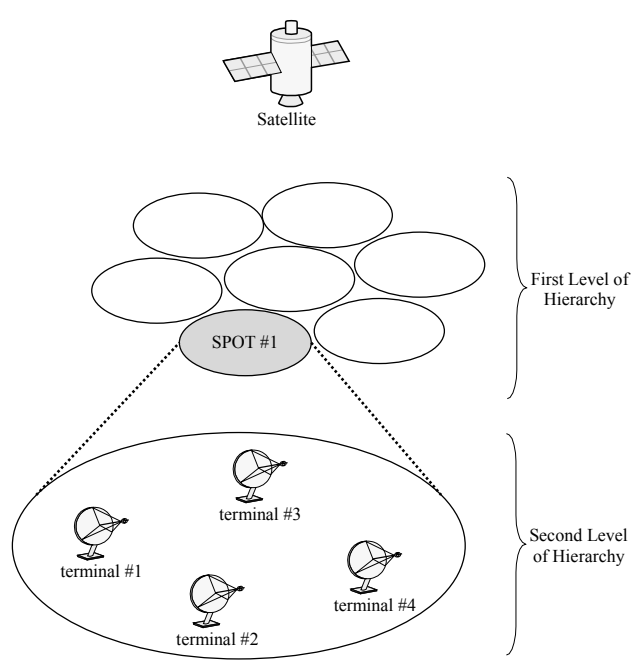

Figure 1. The system architecture.

This two level reference architecture has been considered to make the resource access more flexible and scalable according 
the traffic and the atmospherical conditions of the various beams.

\section{THE FIRST ALLOCATION LEVEL}

In the first level of the system architecture, the bandwidth, available on the satellite to access the return channel, is allocated to the beams. The bandwidth allocation is aimed to optimize the aggregate rate of the system:

$$
\max \sum_{i=1}^{N} f_{\alpha}\left(R_{i}\right)
$$

where $R_{i}$ can be expressed as:

$$
R_{i}=\eta_{B_{i}} B_{i}
$$

In the former equations $N$ is the number of beams defined by the satellite, $R_{i}$ is the rate achieved by the $\mathrm{i}$-th beam with an amount of bandwidth $B_{i}$, while $\eta_{B_{i}}$ is the beam spectral efficiency and it is obtained averaging the spectral efficiency of the satellite terminals located within the borders of the ith beam. The $\eta_{B_{i}}$ coefficient keeps intrinsically into account the average communication channel conditions of each beam, i.e. those beams which are mostly affected by rain events show lower values of $\eta_{B_{i}}$ since the satellite terminals into their borders have low values of the spectral efficiency, due to the stronger channel coding. The $f_{\alpha}(\cdot): \mathbf{R} \rightarrow \mathbf{R}$ is a generic utility function introduced by Mo and Walrand in [7] and here reported:

$$
f_{\alpha}(x)= \begin{cases}\frac{x^{1-\alpha}}{1-\alpha}, & \text { if } \alpha \geqslant 0, \alpha \neq 1 \\ \ln x, & \text { if } \alpha=1\end{cases}
$$

The function $f_{\alpha}$ depends on the fairness parameter $\alpha$ which establishes the degree of fairness used to share the resource. The optimization in (1) is computed in a centralized way, i.e. by the NCC or by a dedicated on-board subsystem. Two possible allocation methods will be studied: a first that is fair, and a second one that is more opportunistic. In the fair method the value of $\alpha$ is 1 and this corresponds to the concept of proportional fairness presented in [8]. In the opportunistic case instead $\alpha=0$ that means no fairness; this value of the fairness parameter, in constrained optimization problems, is equivalent to global optimization. To both the formulations of the optimization problem have been added some constraints:

$$
\begin{array}{ll}
\sum_{i=1}^{N} B_{i} \leqslant B_{\text {sat }}, & \\
B_{i} \geqslant B_{\text {min }_{i}}, & i \in\{1, \ldots, N\} \\
B_{i} \leqslant B_{\text {max }_{i}}, & i \in\{1, \ldots, N\}
\end{array}
$$

The former constraints are considering the satellite available bandwidth, $B_{\text {sat }}$, limitation, the minimum amount of band, $B_{\min _{i}}$, necessary to the beam i-th, and finally the maximum value, $B_{\max _{i}}$, the i-th beam can achieve.

The formulation adopted involves, when opportunistic allocation is applied, the satellite bandwidth is assigned firstly to those beams that shows the higher values of $\eta_{B_{i}}$; instead when fair allocation is performed, the higher $\eta_{B_{i}}$ coefficients weight is made milder by logarithmic utility functions, so those beams affected temporarily by rain events get more bandwidth than in the opportunistic case.

\section{THE SECOND ALLOCATION LEVEL}

In the second level of the hierarchy the satellite terminals located in a beam compete for the bandwidth formerly assigned in the first level. The competition between terminals for the resource is modeled using the game theory. In such competition the aim of each terminal is to achieve as much bandwidth as possible in order to transmit on the return channel at the highest possible throughput. The satellite terminal throughput, $T_{s t}$, has been related with the occupied bandwidth, $B$, through the following relation:

$$
T_{s t}=p_{i b} r_{c} \frac{2}{1+\rho}\left[1-P_{e}\left(\gamma_{r}\right)\right]^{L} B
$$

where $p_{i b}$ is the percentage of information bits on the total in the traffic burst containing one ATM cell, $r_{c}$ is the channel coding rate in use, $\rho$ is the roll-off factor, $P_{e}$ is the bit error probability function of the signal-to-noise ratio $\gamma_{r}$ and $L$ is the number of bits of a single traffic burst.

Two different game theoretical frameworks have been developed: the first is based on the non-cooperative game approach, while the second one has been formulated using the cooperative game approach. The structure of the game has been defined for both the cases and it is basically the same: the satellite terminals are the players of the game, the strategies are constituted by the different amounts of bandwidth a terminal can choose to occupy. As far as it concerns about the utility functions, for the non-cooperative framework logarithmic functions of the throughput have been used, while for the cooperative case linear utility functions have been defined.

The two alternatives will be compared in order to establish which one is more performing in case of heavy channel attenuations due to rain events.

\section{A. Non-cooperative Framework}

The non-cooperative framework adopts the n-person concave game model theorized by Rosen in [9]. The n-person concave game model has the property, under specific conditions, to have a Nash Equilibrium Point that is unique as well. At Nash Equilibrium the players adopt the best possible answer according the other players strategies, i.e. no one achieves gain deviating from the equilibrium point. Considering our study case, the Nash Equilibrium Point, $\mathbf{B}^{*}$, is formally defined in the following way:

$$
\varphi_{j}\left(B_{j}^{*}, \mathbf{B}_{-\mathbf{j}}^{*}\right) \geqslant \varphi_{j}\left(B_{j}, \mathbf{B}_{-\mathbf{j}}^{*}\right), \quad \forall B_{j} \in S_{j}
$$

where $\varphi_{j}(\cdot)$ is the utility function for the $\mathrm{j}$-th user, $B_{j}^{*}$ is the best bandwidth occupation strategy for the user $\mathbf{j}$-th, $\mathbf{B}_{-\mathbf{j}}^{*}$ is the vector of other players best answer strategies and $S_{j}$ is the strategies feasible set for the j-th player. The set $S$, product of 
the each player sets $S_{j}$, is a convex and compact subspace of $\mathbf{R}^{M}$, where $M$ is the number of players. This fact is essential to grant the existence of a Nash Equilibrium. Such equilibrium point is also unique since the utility functions used are strictly concave.

The Nash Equilibrium has been found using a gradient projection algorithm, as suggested in [9]:

$$
\mathbf{B}^{k+1}=\mathbf{B}^{k}+\alpha^{k}\left(\overline{\mathbf{B}}^{k}-\mathbf{B}^{k}\right)
$$

with $\overline{\mathbf{B}}^{k}$ so defined:

$$
\overline{\mathbf{B}}^{k}=\left[\mathbf{B}^{k}+\tau^{k} \mathbf{g}\left(\mathbf{B}^{k}\right)\right]^{+}
$$

In the former ones, $\mathbf{B} \in S$ is the vector containing the bandwidth allocations for each satellite station, $\alpha^{k} \in[0,1]$ is a scalar coefficient that force the bandwidth allocation vector to stay in the feasible set $S, \tau^{k}$ is the algorithm stepsize, $\mathbf{g}\left(\mathbf{B}^{k}\right)$ is the pseudogradient of $\mathbf{B}$ and the operator $[\cdot]^{+}$does the projection of its argument on the set $S$.

Each terminal computes the vector $\mathbf{B}^{*}$ solution of the game in a distributed way, on the basis of common information shared by the terminals of a beam. The common knowledge between satellite terminals is mandatory to make the equilibrium point reachable.

\section{B. Cooperative Framework}

The second level cooperative framework is based on the cooperative games concepts introduced by Nash in [10]. In a cooperative game the players don't have purposes neither totally coincident nor totally opposed. However it's supposed a forcing agreement can be reached by the players of the game through a reciprocal information exchange. Assuming also the players cannot group into coalitions, the cooperative problem can be analyzed as an n-persons bargaining problem similar to the one theorized in [11]. For these kind of problems, the solution is achieved maximizing the productory of the utility funtions. In the cooperative framework the utility functions have been considered linear respect to the throughput, so the problem becomes:

$$
\max \prod_{j=1}^{M}\left[\left(T_{s t_{j}}-T_{s t-\min _{j}}\right)+1\right]
$$

where $M$ is the number of players involved in the game, $T_{s t_{j}}$ the throughput for the $\mathrm{j}$-th player and $T_{s t-m_{i n}}$ is the minimum throughput requested, and granted, to the $\mathrm{j}$-th terminal. For this kind of problem the solution is the Nash Bargaining Point, NBP. Such point has the property to be Pareto optimal, i.e. the bandwidth strategies maximizing (11) achieve the best possible results for the players, unlike the Nash Equilibrium Point that generally isn't Pareto efficient. This is the actual difference between the two solution points. The (11) can be transformed into:

$$
\max \sum_{j=1}^{M} \ln \left[\left(T_{s t_{j}}-T_{s t-\min _{j}}\right)+1\right]
$$

which is equivalent to the original problem since the feasibility set is convex and compact, and the utility functions are injective and strictly concave. The problem (12) has been solved using convex programming methods as Lagrange multipliers method.

\section{Computer Simulations and Results}

The whole system has been implemented in Matlab $\mathbb{R}$ environment and simulations have been performed in order to analyze and compare the allocation methods of the two levels hierarchy. The DVB-RCS specifications, [2] and [12], have been considered to define the satellite network physical layer and the traffic burst format. Table I contains the other parameters used in the simulations.

Table I

SYSTEM PARAMETERS

\begin{tabular}{|c|c|}
\hline Parameter & Value \\
\hline \hline Uplink transmission power & $0 \mathrm{dBW}$ \\
\hline Antenna gain & $45 \mathrm{~dB}$ \\
\hline Distance satellite-terminal & $38000 \mathrm{~km}$ \\
\hline Uplink frequency & $29.7 \mathrm{GHz}$ \\
\hline Pointing loss attenuation & $1 \mathrm{~dB}$ \\
\hline G/T factor of satellite receiver & $13 \mathrm{~dB} / \mathrm{K}$ \\
\hline
\end{tabular}

A single traffic class has been assumed while the traffic packets incoming in the terminals queues have been modeled with a Poisson distribution. The attenuation term due to rain has been modeled using a lognormal distribution.

The first level allocation methods have been compared in terms of the average rate of the beams. The comparisons have been done varying different parameters as the satellite available bandwidth, the system beams number, the percentage of beams experimenting rain. In figure 2 the beam average rate is plotted as function of the satellite available uplink bandwidth. It's evident the increasing difference there is between the two methods for high amounts of bandwidth, instead, when the band is lacking, both the opportunistic and fair method achieve the same performances. The average rate as function of the beams number it is shown in figure 3. The trend of both the curves is decreasing in a polynomial way with the number of beams growing. As in the former graph, also in this one it's evident the opportunistic allocation curve it's above the fair allocation curve till the number of beams is under the value of 35 . The two methods are equivalent in terms of rate when the number of beams exceeds the value of 35 . In figure 4 is graphed the rate as function of the percentage of beams affected by rain. In this graph when rain events affects more than the $35 \%$ of system beams, the fair allocation line crosses the opportunistic one. This fact implies the fair method is better in such situations.

The two game theoretical frameworks used in the second level will be now considered. Unlike the first level, the average throughput of satellite terminals will be examined in the second level. The variables in this case are the number of 


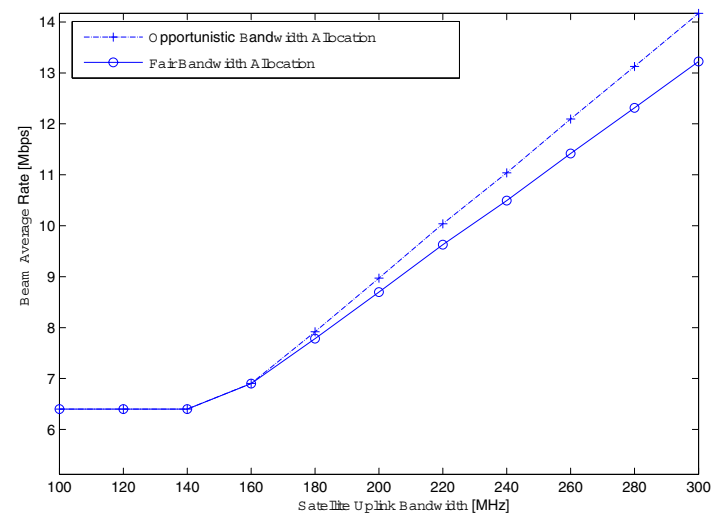

Figure 2. Average rate as function of the satellite uplink bandwidth.

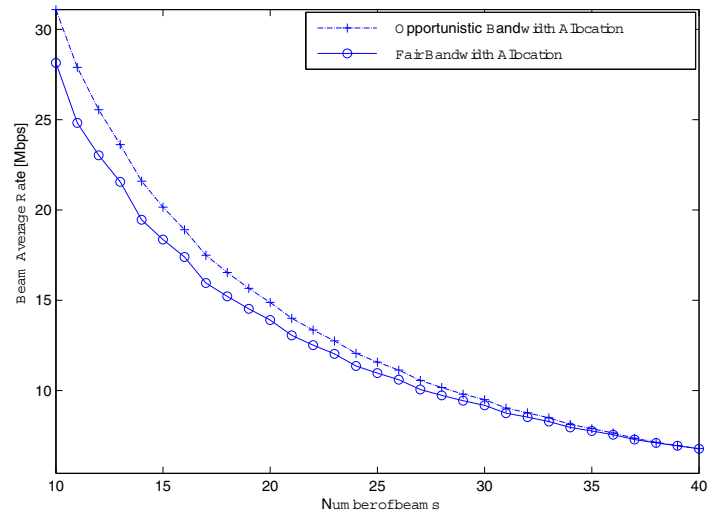

Figure 3. Average rate as function of the system number of beams.

terminals in a beam and the percentage of terminals experimenting attenuation due to rain. In figure 5 e 6 are shown the trends of average throughput in two different kinds of beam: a first affected by rain attenuation and a second one which isn't. With "beam affected by rain attenuation" is meant a beam in which all terminals experiment this kind of attenuation. For the case of attenuated beam, figure 5, the comparison between the frameworks reveals that the cooperative based allocation achieves better throughput performance. The co-

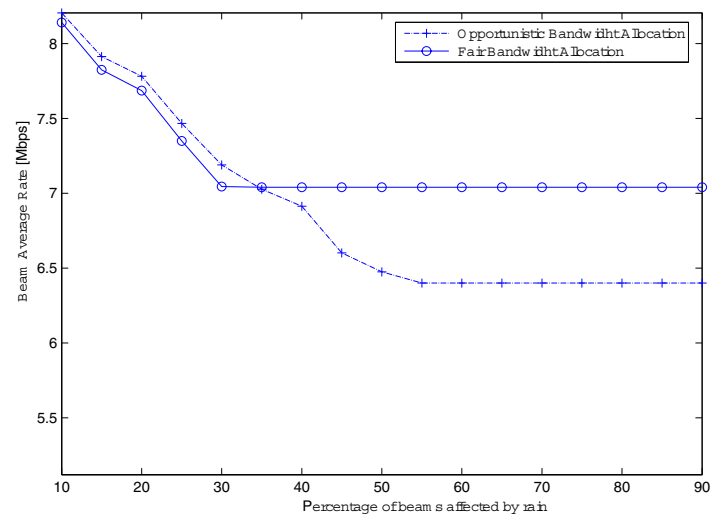

Figure 4. Average rate as function of beams percentage affected by rain.

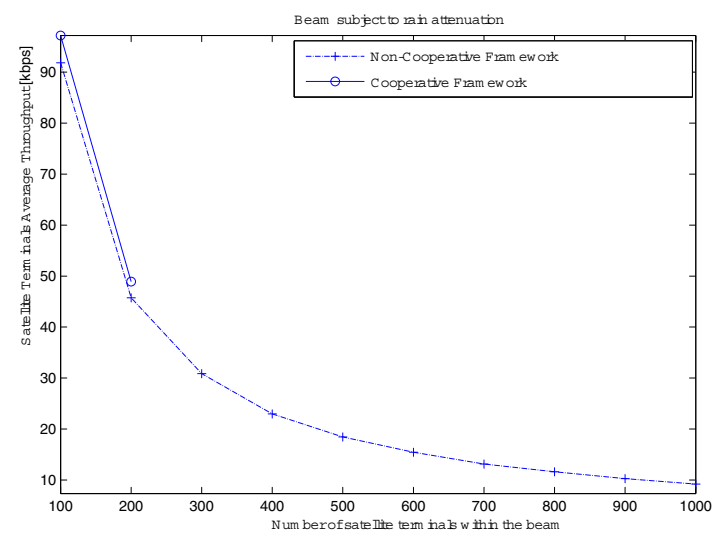

Figure 5. Average throughput as function of the number of terminals in the beam.

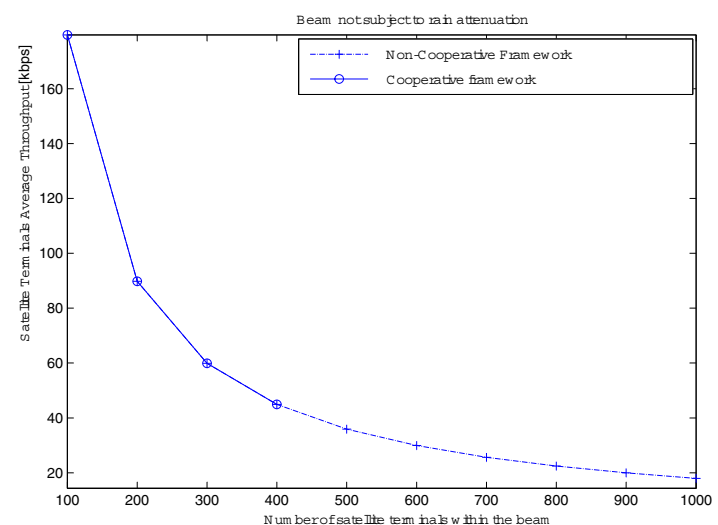

Figure 6. Average throughput as function of the number of terminals in the beam.

operative framework has a drawback, it cannot be used in contests where the number of terminals is greather than 200, that is coeherent with the formulation adopted. Differently the non-cooperative framework is always valid, due to the actual competitions occurring between terminals. In the case of the beams not subject to rain attenuation, figure 6 , both the frameworks achieve identical performances as it is shown by the two overlapping lines. In the graph it is also evident the limitation of the cooperative framework as in the former case. The graph in figure 6 shows a comparison between the two frameworks. The percentage of terminals experimenting rain events has been considered as varying parameter. Two aspects can be identified: the cooperative framework achieve always better throughput values than the non-cooperative allocation. Moreover it's evident the difference between the approaches increases costantly when the percentage value tends to $90 \%$.

\section{CONCLUding REMARKS}

A two level hierarchical system architecture based on DVBRCS has been developed. The first level adopted two different allocation methods: opportunistic and fair. These methods have been compared through simulations with Matlab. It has been found that, in normal conditions, the opportunistic allocation 


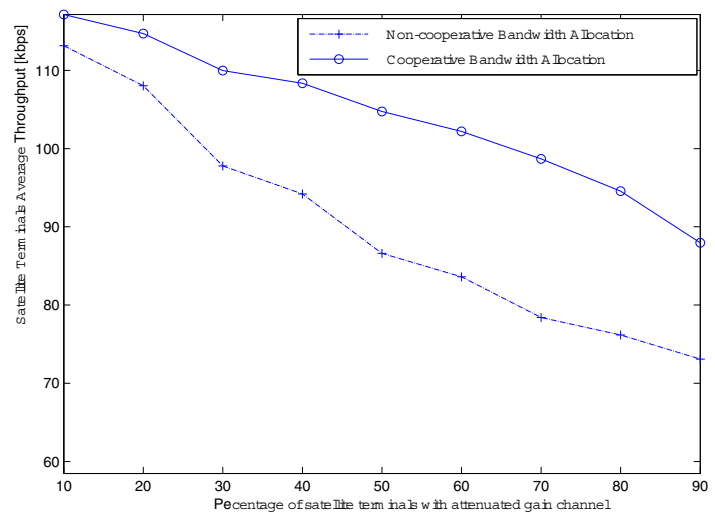

Figure 7. Average throughput as function of the percentage of terminals affected by rain.

achieves better results in terms of beam average rate. In presence of rain events affecting a certain percentage of beams, with fair allocation it's reached an higher average rate respect to the opportunistic case.

As far as it concerns the second level resource allocation the simulations have shown that the non-cooperative and cooperative game theory based allocations produce identical results in normal conditions. Instead for the case of a beam experimenting rain attenuation the cooperative framework has been found to achieve higher throughput values than the noncooperative one. A limitation due to the formulation emerged in the cooperative framework. Such limitation suggests cooperative framework could be suitable to manage the second level available bandwidth for privileged traffic connections, as high priority elastic connections, in both situations.

\section{ACKNOWLEDGMENT}

This work has been carried out in the framework Satellite Network of Excellence (SatNEx), found by European Commission in the $6^{\text {th }}$ Frame Programme. For more info: www.satnex.org.

\section{REFERENCES}

[1] ETSI, Digital Video Broadcasting (DVB); Second generation framing structure, channel coding and modulation systems for Broadcasting, Interactive Services, News Gathering and other broadband satellite applications, EN 302 307, Rev. 1.1.2, Jun. 2006.

[2] - Digital Video Broadcasting (DVB); Interaction channel for satellite distribution systems, EN 301 790, Rev. 1.4.1, Apr. 2005.

[3] M. Á. Vázquez Castro, L. Albiol, J. Alins, J. Cebrián, J. Mata, A. Morell, C. Morlet, G. Seco Granados, and F. Vieira, "Ip-friendly cross-layer optimization of DVB-S2/RCS," in Proc. of the 9th International Workshop on Signal Processing for Space Communications, Sep. 2006.

[4] H. Yaïche, R. R. Mazumdar, and C. Rosenberg, "A game theoretic framework for bandwidth allocation and pricing in broadband networks," IEEE/ACM Transactions on Networking, vol. 8, no. 5, pp. 667-678, Oct. 2000.

[5] Z. Fang and B. Bensaou, "Fair bandwidth sharing algorithms based on game theory frameworks for wireless ad-hoc networks," in Proc. of Twenty-third Annual Joint Conference of the IEEE Computer and Communications Societies, INFOCOM '04, vol. 2, Mar. 2004, pp. 1284 1295.

[6] A. B. MacKenzie and S. B. Wicker, "Game theory in communications: motivation, explanation, and application to power control," in Proc. IEEE Global Telecommunications Conference, GLOBECOM '01, vol. 2, Nov. 2001, pp. 821-826.
[7] J. Mo and J. Walrand, "Fair end-to-end window-based congestion control," IEEE/ACM Transactions on Networking, vol. 8, no. 5, pp. 556567 , Oct. 2000.

[8] F. Kelly, "Charging and rate control for elastic traffic," European Transactions on Telecommunications, vol. 8, pp. 33-37, Jan. 1997.

[9] J. B. Rosen, "Existence and uniqueness of equilibrium points for concave n-person games," Econometrica, vol. 33, no. 3, pp. 520-534, Jul. 1965.

[10] J. F. Nash, "Two-person cooperative games," Econometrica, vol. 21, no. 1, pp. 128-140, Apr. 1953.

[11] — , "The bargaining problem," Econometrica, vol. 18, no. 2, pp. 155162, Apr. 1950

[12] ETSI, Digital Video Broadcasting (DVB); Interaction channel for satellite distribution systems; Guidelines for the use of EN 301 790, TR 101 790, Rev. 1.3.1, Sep. 2006 